induction therapy was more common in severe patients $(71.8 \%$ of patients having at least one comorbidity) compared to mild patients (55.1\%). Since BVAS was not measured routinely, clinical response was categorized as full (no vasculitis activity and GC taper on track), partial (reduction in vasculitis activity and major organ damage arrested) and no response (no improvement in vasculitis). Clinical response is presented below (\% patients) for combination of incident and relapsing patients demonstrating that response varied with many patients having slow and/or incomplete response. Response varied by severity of the disease when induction therapy commenced.

Conclusion: Incident and relapsing AAV patients have variable disease severity at the time of induction therapy. Response to induction therapy is with few exceptions better in patients with milder AAV but overall many patients are slow to respond or have only a partial response to current induction therapy.

Acknowledgement: This work was supported by Vifor Pharma

Disclosure of Interests: Peter Rutherford Employee of: Vifor Pharma, Dieter Götte Employee of: Vifor Pharma

DOI: 10.1136/annrheumdis-2019-eular.1959

\section{THU0316 PROTEINASE-3 REGULATING MICRO-RNA IN GRANULOMATOSIS WITH POLYANGIITIS}

Susanne Schinke ${ }^{1}$, Nick Reichard ${ }^{2}$, Barbara Russo $^{3}$, Antje Müller $^{2}$, Martin Laudien ${ }^{4}$, Robert Häsler ${ }^{5}$, Gabriela Riemekasten ${ }^{1}$, Peter Lamprecht ${ }^{1}$. ${ }^{1}$ University Lübeck, UKSH Campus Lübeck, Rheumatology and Clinical Immunology, Lübeck, Germany, ${ }^{2}$ University Lübeck, Lübeck, Germany; ${ }^{3}$ University Hospital and School of Medecine, Immunology and Allergy, Geneva, Switzerland; ${ }^{4}$ UKSH Campus Kiel, HNO-Klinik, Kiel, Germany; ${ }^{5}$ Christian-Albrechts-University Kiel, Clinical Molecular Biology, Kiel, Germany

Background: Dysregulated miRNA expression profiles have been described in diverse chronic inflammatory diseases. We previously did a microarray screening of 847 miRNAs in nasal tissue from GPA patients and we found a disease associated alteration of miRNA expression compared to healthy controls $(\mathrm{HC})$ and chronic rhinosinusitis (CRS)

Objectives: In order to identify new miRNA targets of potential pathophysiological relevance in GPA, we validated dysregulated miRNAs by qPCR
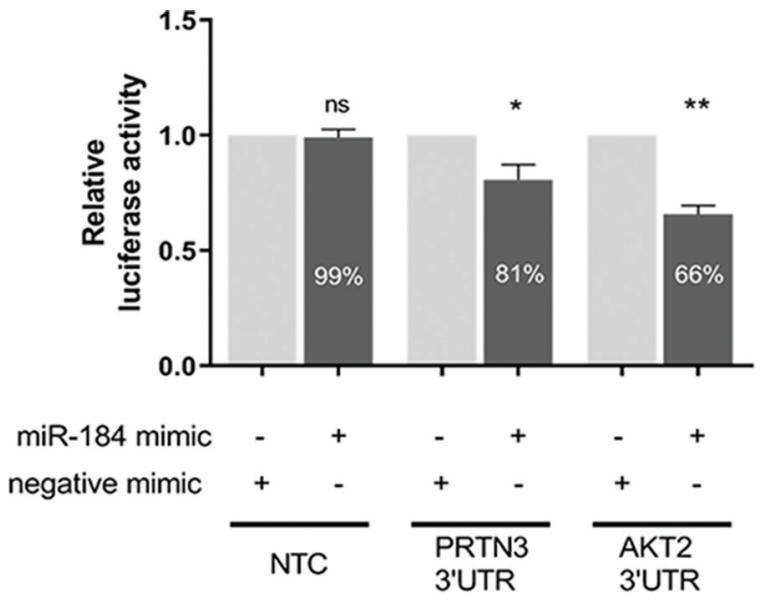

Fig. 1: Dual-luciferase reporter assays showing a decrease in luciferase activity when fused to the 3'UTR of PRTN3 3 and co-transfected with microRNA-184 mimic. No reduction can be observed for the pmirGLO reporter without inserted 3'UTR (NTC). The 3'UTR of AKT2 was used as a positive control, since it is a validated target of miR-184!. Effects of miR-1\$4 targeting the reporter was compared to co-transfection of reporter with cel-miR-67 (negative mimic). Data represent 3 independent experiments with triplicate measurements. P values were calculated by one sample t-test. $* \mathrm{p}<0.05 ;{ }^{*} \mathrm{P}<0.005 ; \mathrm{ns}=$ not significant; error bars display standard deviation.

Abstract THU0316 - Figure 1. in GPA nasal tissue biopsies and sera. Moreover, we screened GPA associated miRNAs for their potential to regulate proteinase-3 (PRTN3).

Methods: In an independent validation cohort (tissue and sera from 14 GPA-patients, 10 disease controls: CRS and Crohn's/CD) 12 miRNAs were examined by GPCR. Validated and computational miRNA targets were identified by mirDIP algorithms. The inhibitory capacity of miRNAs on Proteinase-3 (PRTN3) was estimated by a dual-luciferase reporter system $\left(\right.$ Promega ${ }^{\circledR}$ ). The 3'UTR-PRTN3 sequence was cloned and inserted into the pmirGlo vector and co-transfected with the hsa-mirna mimics $\left(\right.$ Dharmacon ${ }^{\circledR}$ ) into HeLa cells. As a second method, the effect of miR184 transfection on the endogenous PRTN3 expression in the human myeloid leukemia cell line HL-60 was estimated by western blot.

Results: Microarray screening revealed alterations of 24 miRNAs in GPA nasal tissue compared to $\mathrm{HC}$ and CRS. qPCR confirmed dysregulation of 6 tissue related miRNAs also in GPA sera. Compared to CD 4 miRNAs (miR-10b, -99a/100, -125b, -532-3p) were down regulated in GPA tissue. The miRNA with the highest expression level in nasal tissue from GPA was miR-184. miR-184 along with miR-708 and mir-214-5p were also predicted to target PRTN3 by the mirDIP algorithm. The dual-luciferase reporter assay revealed a significant reduction of PRTN3 expression by miR-184, while these effects could not be observed for miR-708 or miR 214-5p. The transfection of miR-184 into $\mathrm{HL}-60$ cells resulted in a dosedependent knockdown of PRTN3 expression as detected by Western blot Conclusion: Characteristic miRNA signatures in GPA, CRS and CD suggest distinct pathophysiological mechanisms. It indicates at a local miRNA dysregulation in inflamed GPA tissue with a corresponding serum signature that might serve as novel biomarkers. To our knowledge this is the first analysis that attempts to correlate GPA-associated miRNA expression patterns in tissue with serum. Moreover, this is the first description of a miRNA (miR-184) that potentially regulates the expression of the GPA autoantigen PRTN3.

\section{REFERENCES:}

[1] O'Connell $R$ et.al. Physiological and pathological roles for microRNAs in the immune system. Nat Rev Immunol. 2010;10(2):111-122.

[2] Fasseu $\mathrm{M}$ et al. Identification of restricted subsets of mature microRNA abnormally expressed in inactive colonic mucosa of patients with inflammatory bowel disease. PLoS One. 2010 Oct 5;5(10).

[3] Neudecker, V. MicroRNAs in mucosal inflammation. J Mol Med (Berl) 2017 September; 95(9): 935-949

[4] Coit $P$ et al. An update on the role of epigenetics in systemic vasculitis. Curr Opin Rheumatol. 2018; 30(1): 4-15

Disclosure of Interests: Susanne Schinke Grant/research support from: travel and congress expenses from different companies pfizer, ucb, chemocentryx, Janssen-Cilag, msd, Nick Reichard: None declared, Barbara Russo: None declared, Antje Müller: None declared, Martin Laudien Paid instructor for: Olympus, Speakers bureau: Novartis, Robert Häsler: None declared, Gabriela Riemekasten Consultant for: Chugai, F. Hoffmann-La Roche, Speakers bureau: Chugai, F. Hoffmann-La Roche, Peter Lamprecht: None declared DOI: 10.1136/annrheumdis-2019-eular.4575

\section{THU0317 IDENTIFICATION OF ENDOTHELIAL PROTEIN C RECEPTOR AND SCAVENGER RECEPTOR CLASS B TYPE 1 AS MAJOR AUTOANTIGENS IN TAKAYASU ARTERITIS}

Tsuyoshi Shirai, Tomoyuki Mutoh, Tomonori Ishii, Hiroshi Fujii, Hideo Harigae. Tohoku University Graduate School of Medicine, Sendai, Japan

Background: Takayasu arteritis (TAK) affects the aorta and its major branches. It has been recognized that high numbers of patients with TAK possessed antiendothelial cell antibodies (AECA), which have potential to induce vascular lesion. However, their major target antigens remain unclear. The target antigens of AECA are plasma membrane proteins, and traditional methods to identify autoantigens do not differentiate between cell-surface molecules and intracellular molecules. To overcome this problem, we have developed an expression cloning system to identify cell-surface antigens: serological identification system for autoantigens using a retroviral vector and flow cytometry $(\mathrm{SARF})^{1-3}$. Because there 